\title{
Gel Electrophoretic Studies on Proteoglycans and Collagen of Abnormal Human Growth Cartilage: Proteoglycan Abnormalities in Pseudoachondroplasia and in Kniest's Disease
}

\author{
V. STANESCU $(19)$ AND P. MAROTEAUX \\ Unite de Recherches de Génétique Médicale, INSERM, Hôpital des Enfants-Malades, Paris, France
}

Extract

The microchemical study of growth cartilage biopsies may improve the classification and the genetic advice of some types of growth disturbances and contribute to the understanding of biochemical defects.

Small tibial growth cartilage biopsies were performed during orthopedic surgery in cases with achondroplasia, pseudoachondroplasia (three cases), Kniest's disease (two cases), diastrophic dwarfism (two cases), parastrematic dwarfism, pycnodysostosis, mucolipidosis type III. Blount's disease, and in three normal growing children. Five human fetal cartilages were also studied.

The proteoglycans were extracted with $4 \mathrm{M}$ guanidinium chloride. After dialysis against $8 \mathrm{M}$ urea at $\mathrm{pH} 7$, the proteoglycans were obtained by ion chromatography in urea on DEAE-cellulose and submitted to gel electrophoresis on polyacrylamide-agarose gels. The gel electrophoresis of the proteoglycans of growth cartilage of normal growing children gave two metachromatic bands situated close one to another. The proteoglycans extracted from fetal growth cartilage gave a single band with a slightly slower migration. An abnormal gel electrophoretic pattern was found in pseudoachondroplasia and in Kniest's disease. In pseudoachondroplasia a single wide band was found; in overcharged tubes several thin, more rapid bands appeared in addition to the main band. In Kniest's disease three bands were found.

In all of the other syndromes studied two normally or almost normally situated bands were present. Small differences in the width and intensity of the bands observed in several cases were difficult to assess. In all cases except mucolipidosis III and Kniest's disease the collagen was extracted by using limited cleavage and solubilization with pepsin, purified and analyzed by polyacrylamide gel electrophoresis. A type of collagen with a single $\alpha$ band was found.

\section{Speculation}

Pseudoachondroplasia and Kniest's disease are due to genetic alterations of proteoglycans. These abnormalities produce the growth disturbance and the skeletal dysplasia. It is likely that the alteration is limited to cartilage or to cartilage and bone. The abnormal endoplasmic reticulum found in both diseases suggests defects in synthesis and/or transfer of proteoglycans or of their precursors. However, the possibility of a different primary defect affecting secondarily the gel electrophoretic pattern of extracted proteoglycans could not be excluded.

The histochemical and microchemical study of growth cartilage biopsies may improve the classification and the genetic advice of some types of growth disturbances. The data obtained might also contribute to the understanding of the pathogenesis of the syndromes investigated. In previous studies we found abnormalities of glycosaminoglycans and collagen in some types of abnormal human growth cartilage $(11,14)$. In the present study a gel electrophoretic analysis of proteoglycans and collagen extracted from normal and abnormal human growth plate biopsies was performed.

\section{MATERIAL AND METHODS}

\section{CASE MATERIAL}

The proximal tibial growth cartilage of 13 cases with growth disturbances was studied (Table 1). Three normal growing children aged 5,7 , and 12 years were studied as control subjects. Five fetal specimens selected from cases of therapeutic abortion and obtained within $10 \mathrm{~min}$ of the abortion were also studied. The gestional age of the fetuses was determined from the crown-rump length.

\section{BIOPSY PROCEDURE}

In all the cases (except the fetuses) the cartilage was obtained by biopsy performed during orthopedic surgery, according to a previously described procedure (II).

\section{TISSUE PREPARATION}

The biopsy fragments and the fetal specimens were quickly frozen with carbon dioxide and stored at $-60^{\circ}$. Undecalcified frozen sections were cut in a cryostat at $-20^{\circ}$. Groups of $40 \mu \mathrm{m}$ thick sections for microchemical determinations were alternated with groups of sections $14 \mu \mathrm{m}$ thick to be used for histochemical staining. The $40-\mu \mathrm{m}$ thick sections were freeze-dried at $-40^{\circ}$ for 4- 5 days at a vacuum of about $0.01 \mathrm{~mm} \mathrm{Hg}$. The cartilage was separated from bone by microdissection of the freeze-dried sections under a binocular dissecting microscope.

\section{GEL ELECTROPHORETIC ANALYSIS OF PROTEOGLYCANS}

The microdissected lyophilized sections were extracted with $4 \mathrm{M}$ guanidinium chloride, $0.05 \mathrm{Tris}-\mathrm{HCl}, \mathrm{pH} \mathrm{7.2,} \mathrm{at} 4^{\circ}$ with magnetic stirring for $48 \mathrm{hr}(10)$.

After dialysis against $8 \mathrm{M}$ urea in Tris- $\mathrm{HCl}$ buffer, $0.05 \mathrm{M}, \mathrm{pH}$ 7.0. at $4^{\circ}$ (Dia-Flo pressure cell, Amicon, UM 20E membrane) the proteoglycans were obtained by ion chromatography on a DEAE-cellulose column in the $2 \mathrm{M} \mathrm{NaCl}$ fraction $(1,2)$. This fraction was dialyzed against the $8 \mathrm{M}$ urea in Tris- $\mathrm{HCl}$ buffer, $\mathrm{pH}$ 7.0 solution, to decrease the $\mathrm{NaCl}$ concentration to $0.02 \mathrm{M}$. The proteoglycans were subjected to gel electrophoresis on large pore composite polyacrylamide-agarose gels. The method of McDevitt and Muir (5), modified slightly $(1.2 \%$ acrylamide and $0.7 \%$ agarose), was used. Samples of proteoglycans equivalent to $1.5-2.5$ $\mu \mathrm{g}$ hexuronic acid were layered on each tube. The electrophoresis was performed in a cold room with a current of $5 \mathrm{ma} / \mathrm{tube}$ and a voltage gradient of about $20 \mathrm{~V} / \mathrm{cm}$, the duration of the run being about $60 \mathrm{~min}$. The gels were stained with $0.2 \%$ toluidine blue in 0.1 $\mathrm{N}$ acetic acid and the excess dye was removed with $0.1 \mathrm{~N}$ acetic 
Table 1. Study of proximal tibial growth cartilage

\begin{tabular}{lc}
\multicolumn{1}{c}{ Case } & Age. yr \\
\hline Pseudoachondroplasia & $117 / 2$ \\
Pseudoachondroplasia & $76 / 12$ \\
Pseudoachondroplasia & 7 \\
Kniest's disease & 8 \\
Kniest's disease & 10 \\
Achondroplasia & 11 \\
Diastrophic dwarfism & 8 \\
Diastrophic dwarfism & 10 \\
Blount's disease & 13 \\
Pycnodysostosis & 13 \\
Parastrematic dwarfism & 9 \\
Mucolipidosis type III & 14 \\
Multiple exostoses & 10 \\
Normal & 5 \\
Normal & 7 \\
Normal & 12 \\
Fetuses (5 cases) & $15-19^{1}$ \\
\hline
\end{tabular}

${ }^{1}$ Weeks of gestational age.

acid, followed by washings of water. A sample of chondroitin sulfate was included in all runs as a reference standard. Two tubes of each sample were analyzed and runs were repeated.

\section{GEL ELECTROPHORETIC ANALYSIS OF COLLAGEN}

After the extraction of the major part of proteoglycans the cartilage was dissolved by using the limited cleavage and solubilization with pepsin technique according to the method of Miller (6). After incubation the digestion mixture was clarified by centrifugation and the collagen was precipitated by addition of sodium chloride. The precipitate was retrieved by centrifugation, redissolved in $1 \mathrm{M}$ sodium chloride, $\mathrm{pH} 7.5$, and dialyzed against a large volume of $0.05 \mathrm{M}$ acetic acid. Further purification was performed using the method of Miller et al. (7). The material obtained was analyzed by polyacrylamide gel electrophoresis according to Stark and Kühn (15). Samples of collagen extracted and purified by the same method from the skin and cartilage of a baboon were included as controls. Acid-soluble collagen from rat tail tendon was also analyzed in the same run.

\section{RESULTS}

\section{PROTEOGLYCANS}

The gel electrophoresis of the proteoglycans of growth cartilage of normal growing children yielded two metachromatic bands situated close one to another. The proteoglycans extracted from fetal growth cartilage gave a single band with a slightly slower migration (Fig. 1). The chondroitin sulfate standard gave a single band which migrates faster than the proteoglycans.

An abnormal gel electrophoretic pattern of proteoglycans was found in pseudoachondroplasia and in Kniest's disease.

In psuedoachondroplasia a single wide band was found (Fig. 2). In overcharged tubes several thin and more rapid bands appear in addition to the main wide band (Fig. 3). The pattern is different both from that found in normal children (two bands) and in fetuses (one narrow and slightly delayed band).

In Kniest's disease three bands were found. The third band is faster than the normal bands. The same pattern was found in the two cases studied (Fig. 4).

In all the other syndromes studied two normally or almost normally situated bands were found. Small differences in the wideness and the intensity of the bands observed in several cases are difficult to assess.

\section{COLLAGEN}

The gel electrophoretic analysis of collagen was performed in the normal children and in all of the pathologic cases except the cases with mucolipidosis III and Kniest's disease.

The collagen extracted from the normal children and from the cartilage of the baboon gave a single $\alpha$-chain band. All the abnormal growth cartilages studied gave a single $\alpha$ band with the same migration as the band of normal cartilage. In contrast the collagen extracted from the baboon skin and the acid-soluble collagen from the rat tail tendon yielded two $\alpha$-chain bands (Fig. $5)$.

\section{DISCUSSION}

With the method used the proteoglycans were obtained in highly "dissociative" conditions and no stained material remained at the gel origin. The method of extraction and purification we used in this study yields cartilage proteoglycans with very similar properties (by gel chromatography, distribution in a density gradient, and composition) to the "subunits" (Al-Dl) isolated by the Hascall and Sajdera method (1). However, the existence of dimers or small aggregates (9) could not be excluded. Addition of proteolysis inhibitors does not alter electrophoretic results (13).

The present study demonstrates a definite abnormal gel electrophoretic pattern of "dissociated" proteoglycans obtained from the growth cartilage in two types of chondrodystrophic dwarfs: pseudoachondroplasia and Kniest's disease. This suggests strongly that the genetic defect in these diseases alters the molecular populations of proteoglycans of the growth cartilage. Clinical data suggest that the alteration might be limited to the chondrocytes or to chondrocytes and bone cells. Other connective tissues are probably not or less affected. Further studies are, however, necessary to elucidate this point. The possibility of a different primary defect affecting secondarily the gel electrophoretic pattern of extracted proteoglycans could not be excluded.

Both pseudoachondroplasia and Kniest's disease are spondyloepiphyseal dysplasias with important alterations of the epiphysis and spine, and with severe dwarfism. In pseudoachondroplasia the chondrocytes contain many large vacuoles bound by the endoplasmic reticulum with alternately electron-dense and electron-lucent layers. The vacuoles were observed by Cooper et al. (3) and by us in two of the three cases included in this study in which an ultrastructural examination was performed. In two cases of Kniest's disease included in the present study the ultrastructural investigation disclosed a dilated rough surfaced endoplasmic reticulum cisternae containing a granulo-fibrillar material (13).

In our cases microscopic optical studies on semithin Epon sections and on frozen sections showed that the inclusions of pseudoachondroplasia were positive for protein staining and not metachromatic, whereas the inclusions in Kniest's disease contained metachromatic material (13).

The relationship between the abnormal appearance of the endoplasmic reticulum and the abnormal pattern of the cartilage proteoglycans is still unknown. The abnormal endoplasmic reticulum suggests that the abnormalities of proteoglycans are due to troubles in the synthesis and/or transfer of these compounds or of a factor which plays a role in the formation of small aggregates. The intracellular accumulation of some precursor(s) of a proteoglycan population is one of the possibilities to be considered in pseudoachondroplasia.

It is known that in pseudoachondroplasia the clinical and roentgenologic alterations are not present at birth but become evident during the first or second year of life. This might suggest that the genetic defect does not alter the fetal proteoglycans. It was shown that the gel electrophoretic pattern of growth cartilage proteoglycans is different in fetuses from that found in older animals (12 and the present study). In humans, the change takes place in the first months of extrauterine life (13). An alternative 


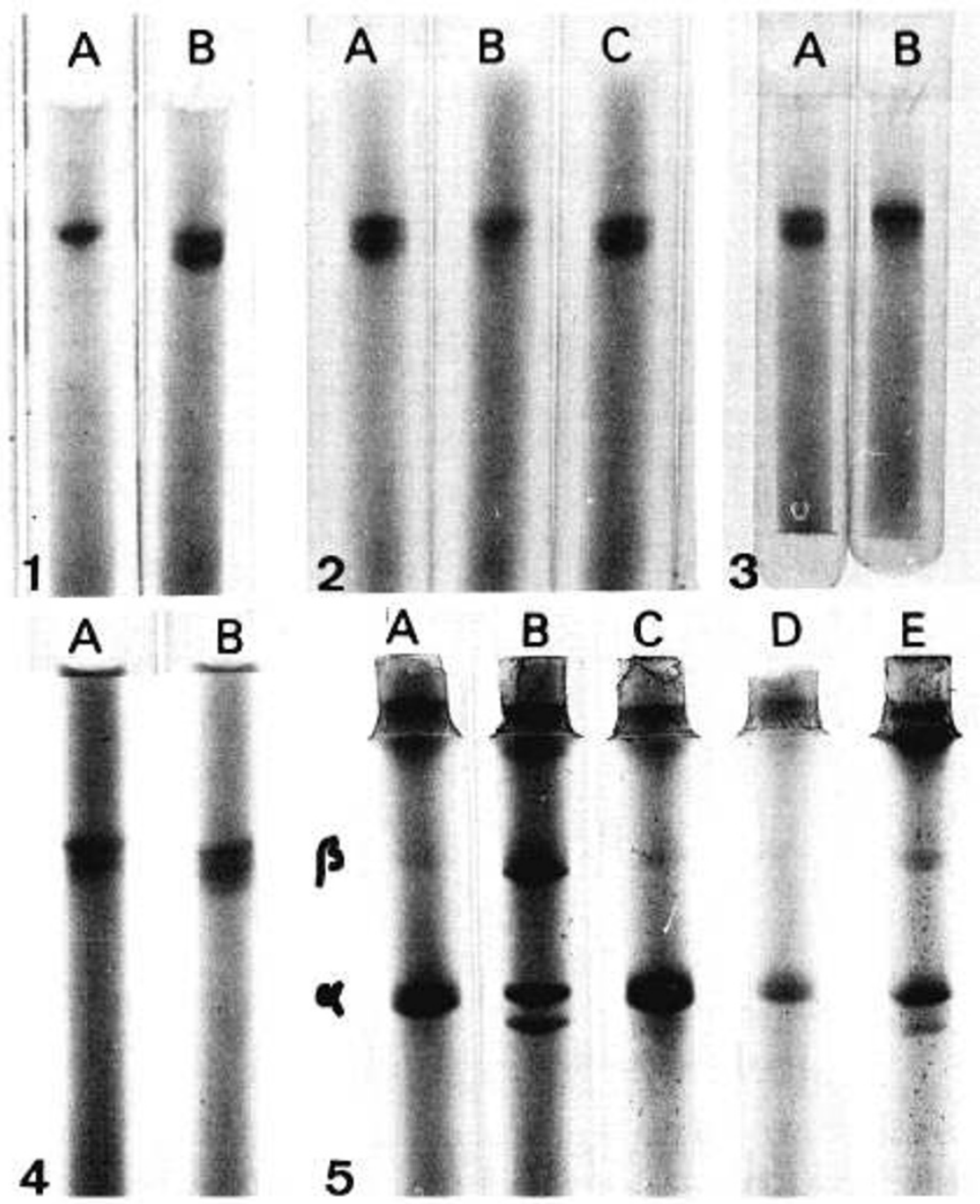

Fig. 1. Proteoglycans. A: fetal proteoglycans; $B$ : Proteoglycans of a 7-year-old child.

Fig. 2. $A$ : normal; $B$ : pseudoachondroplasia; $C$ : diastrophic dwarfism.

Fig. 3. $A$ : normal; $B$ : pseudoachondroplasia, overcharged tube.

Fig. 4. $A$ : normal; $B$ : Kniest's disease.

Fig. 5. Collagen. $A$ : pseudoachondroplasia; $B$ : acid-soluble collagen, rat tendon; $C$ : baboon cartilage; $D$ : diastrophis dwarfism; $E$ : baboon skin.

hypothesis might suggest that the abnormalities become apparent when the child walks and the abnormal cartilage is under stress (3).

Some subtle differences were observed in the gel electrophoretic pattern of proteoglycans in several other syndromes $(e . g .$, differences in the intensity and the width of the normal situated bands or small differences in migration). However, these differences are difficult to assess and the study of additional cases is necessary. On the other hand, identity of position of two bands in our system does not mean necessarily identity of the proteoglycan populations.

The analysis of the small amount of collagen extracted from biopsies of normal and abnormal growth cartilage had a limited purpose. We intended to find out whether abnormal growth cartilage contained collagen displaying two types of chains instead of one. It was found from the literature that in certain circumstances (tissue culture, arthrosic cartilage, preincubation with lysosomal enzymes) $(4,8)$, the chondrocytes produce a significant amount of bone and fibrocyte type of collagen $\left[\alpha_{1(1)}\right]_{2} \alpha_{2}$ in addition or instead of the cartilage type of collagen $\left[\alpha_{1 \text { (II }}\right]_{3}$. In previous histochemical studies we found abnormal fibrosis in the matrix of some pathologic growth cartilages $(11,14)$, and we made the suggestion that the chondrocytes may have an incompletely or abnormal differentiation. However, in all the pathologic cases included in this study, a single $\alpha$ band was found. However, with the method used small amounts of fibrocytic type of collagen might be undetected.

\section{SUMMARY}

The gel electrophoretic pattern of proteoglycans extracted from small biopsies of tibial growing cartilage was studied in cases with achondroplasia, pseudoachondroplasia (three cases), Kniest's disease (two cases), diastrophic dwarfism (two cases), parastrematic dwarfism, pycnodysostosis, mucolipidosis type III, Blount's disease, and multiple exostoses. The results were compared with those obtained in three normal growing children of similar age and in five human fetuses. Gel electrophoresis of proteoglycans showed abnormal patterns in the cases with pseudoachondroplasia and with Kniest's disease. In the same cases, except mucolipidosis III and Kniest's disease, the collagen was analyzed by polyacrylamide gel electrophoresis. In all the cases studied, a cartilage type of collagen with a single $\alpha$ band was found.

\section{REFERENCES AND NOTES}

1. Antonopoulos, C. A., Axelsson, I., Heinegard, D., and Gardell, S.: Extraction and purification of proteoglycans from various types of connective tissue. Biochim. Biophys. Acta, 338: 108 (1974)

2. Antonopoulos, C. A., Engfeldt, B., Gardell, S., and Heinegard, D.: Metabolic 
heterogeneity of proteoglycans from various connective tissues (Abstract). Scand. J. Clin. Lab. Invest. 29 (suppl. 123): 4 (1972).

3. Cooper, R. R., Ponseti, I. V., and Maynard J. A.: Pseudo-achondroplastic dwarfism: A rough-surfaced endoplasmic reticulum storage disorder. J. Bone Joint Surg., 53A: 475 (1973).

4. Deshmukh, K., and Nimni, M. E.: Effects of lysosomal enzymes on the type of collagen synthesized by bovine articular cartilage. Biochem. Biophys. Res. Commun.. 424 (1973)

5. McDevitt, C. A., and Muir, H.: Gel electrophoresis of proteoglycans and glycosaminoglycans on large-pore composite polyacrylamide agarose gels. Anal. Biochem., 44: 612 (1971).

6. Miller, E. J.: Structural studies on cartilage collagen employing limited cleavage and solubilization with pepsin. Biochemistry, ll: 4903 (1972).

7. Miller, E. J., Martin, G. R., Piez, K. A., and Powers, M. J.: Characterization of chick bone collagen and compositional changes associated with maturation. J Biol Chem., 242: 5481 (1967).

8. Nimni, M. E., and Deshmukh, K.: Differences in collagen metabolism between normal and osteo-arthritic human articular cartilage. Science, 181: 751 (1973).

9. Rosenberg. L., Hellmann, W., and Leinschmidt, A. K.: Macromolecular models of proteinpolysaccharides from bovine nasal cartilage based on electron microscopic studies J Biol. Chem. 245: 4123 (1970)

10. Sajdera, S. W., and Hascall, V. C.: Proteinpolysaccharide complex from bovine nasal cartilage: A comparison of low and high shear extraction procedures. $J$
Biol. Chem.. 244: 77 (1969).

11. Stanescu, V., Bona $C_{\text {., }}$ and Ionescu. $V$.: The tibial growing cartilage biopsy in the study of growth disturbances. Acta Endocrinol., 64: 577 (1970).

12. Stanescu, V Maroteaux, P., and Sobczak, E.: Gel electrophoresis of the proteoglycans of the growth and of the articular cartilage from various species. Biomedicine, 19: 460 (1973).

13. Stanescu, R., and Stanescu, V.: Unpublished results

14. Stanescu, V Stanescu, R. and Szirmai, J. A.: Microchemical analysis of the human tibial growth cartilage in various forms of dwarfism. Acta Endocrinol., 69: 659 (1972).

15. Stark. M., and Kuhn, K.: The properties of molecular fragments obtained on treating calfskin collagen with collagenase from clostridium histolyticum. Eur. J. Biochem.. 6: $534(1968)$

16. The technical assistance of Mrs. E. Sobczak and Miss M. P. Richard is gratefully acknowledged.

17. The research contained in the present article was performed with the informed consent of the patients or of the parents of the patients.

18. Portions of this paper were presented at the 22 nd Annual Colloquium on Protides of the Biological Fluids, May 1974. Brugge, Belgium.

19. Requests for reprints should be addressed to: V. Stanescu, M.D., Unité de Recherches de Génétique Médicale. Hôpital des Enfants-Malades, 149, rue de Sèvres, Paris (France).

20. Accepted for publication June 13, 1975

\title{
The Vitamin $\mathbf{B}_{12}$-deficient Rat as a Possible Model of Ketotic Hyperglycinemia
}

\author{
BARBARA O'NEILL ROWLEY, VIRGINIA BROTHERS, AND THEO GERRITSEN ${ }^{(40)}$ \\ Departments of Pediatrics and Physiological Chemistry, Waisman Center on Mental Retardation and Human \\ Development, University of Wisconsin Center for Health Sciences, Madison, Wisconsin, USA
}

\section{Extract}

The rate of oxidation to respiratory $\mathrm{CO}_{2}$ of both carbon 1 of propionate and carbon 1 of glvcine was decreased significantly in vitamin $B_{12}$-deficient rats, to $50 \%$ and $82 \%$ of the control rate, respectively. The activity of the glycine synthase system was reduced during vitamin $B_{12}$ deficiency to $25 \%$ of control activity. Serine hydroxymethyltransferase activity was similar for vitamin $B_{12}$-deficient and control rats. Plasma glycine concentration in vitamin $B_{12}$-deficient rats $(253 \pm 16 \mathrm{nmol} / \mathrm{ml})$ did not differ significantly from that of control rats $(226 \pm 12 \mathrm{nmol} / \mathrm{ml})$. Propionate oxidation was significantly impaired in biotin-deficient rats. However, this impairment, to $66 \%$ of the control rate, was not as large as that generated by vitamin $B_{12}$ deficiency. In contrast to the result obtained in vitamin $B_{12}$-deficient animals, no significant decrease in glycine oxidation could be demonstrated in biotin-deficient animals Plasma glycine concentration of fasted biotin-deficient rats $(339 \pm 26 \mathrm{nmol} / \mathrm{ml})$ did not differ significantly from that of their controls $(371 \pm 32 \mathrm{nmol} / \mathrm{ml})$.

\section{Speculation}

Activity of the glycine synthase system is reduced in both the ketotic and nonketotic forms of hyperglycinemia. The decrease in glycine synthase system activity in vitamin $B_{12}$-deficient rats may be generated by a mechanism similar to that in ketotic hyperglycine - mia, and therefore vitamin $B_{12}$-deficient rats may be useful to study this mechanism.

In 1961, Childs et al. (5) described a patient with a disorder with episodic vomiting, lethargy, ketosis, developmental retardation, and hyperglycinemia and hyperglycinuria. Later studies demonstrated that this disease resulted from an inherited deficiency of propionyl-CoA carboxylase (ATP hydrolyzing) (EC. 6.4.1.3) (13). Similar symptoms may be present in patients with a deficiency in methylmalonyl-CoA mutase (EC. 5.4.99.2) $(17,19)$ and presumed $\beta$-ketothiolase deficiency (acyl-CoA:acetyl-CoA C-acyl transferase, EC. 2.3.1.16) (9). The role of these enzymes in propionate metabolism is shown in Figure 1.

The defect in glycine metabolism in the different forms of ketotic hyperglycinemia appears to be secondary to the impairment in organic acid metabolism. The mechanism of interaction of organic acid metabolism with glycine metabolism is as yet unexplained. The major pathway for glycine degradation in mammals appears to be via conversion to methylenetetrahydrofolate and $\mathrm{CO}_{2}$, catalyzed by the glycine synthase system (EC. 2.1.2.10) (28). The ketotic hyperglycinemia syndrome differs clinically from the metabolic disease, nonketotic hyperglycinemia, a disorder first described by Gerritsen et al. (7). Nonketotic hyperglycinemia is characterized principally by severe mental retardation and caused by an inherited primary defect in the glycine synthase system (26). 